\title{
Shape-based Transfer Functions for Volume Visualization
}

\author{
Jörg-Stefan Praßni* $\quad$ Timo Ropinski ${ }^{\dagger} \quad$ Jörg Mensmann ${ }^{\ddagger} \quad$ Klaus Hinrichs $^{\S}$
}

Visualization and Computer Graphics Research Group (VisCG), University of Münster

\begin{abstract}
We present a novel classification technique for volume visualization that takes the shape of volumetric features into account. The presented technique enables the user to distinguish features based on their 3D shape and to assign individual optical properties to these. Based on a rough pre-segmentation that can be done by windowing, we exploit the curve-skeleton of each volumetric structure in order to derive a shape descriptor similar to those used in current shape recognition algorithms. The shape descriptor distinguishes three main shape classes: longitudinal, surface-like, and blobby shapes. In contrast to previous approaches, the classification is not performed on a per-voxel level but assigns a uniform shape descriptor to each feature and therefore allows a more intuitive user interface for the assignment of optical properties. By using the proposed technique, it becomes for instance possible to distinguish blobby heart structures filled with contrast agents from potentially occluding vessels and rib bones. After introducing the basic concepts, we show how the presented technique performs on real world data, and we discuss current limitations.
\end{abstract}

Index Terms: I.3.7 [Computer Graphics]: Three-Dimensional Graphics and Realism-Color, shading, shadowing, and texture.

\section{INTRODUCTION}

Classification is an essential part of the volume visualization pipeline. By applying transfer functions, the user is able to assign optical properties to individual parts of a volumetric data set. In contrast to a segmentation technique, a classification requires that these individual parts can be distinguished solely based on information present in the data set. The most straightforward classification can be performed by applying 1D transfer functions [19], which use the intensity values stored in the data set to assign optical properties, usually given by an emissive color and an opacity value, to certain subranges within the intensity range. Although 1D transfer functions are easy to use and do not rely on any pre-computation, they have the drawback that they do not allow to discriminate features within a data set which have an overlapping intensity range. To deal with this shortcoming, multidimensional transfer functions have been proposed [14]. 2D transfer functions based on intensity gradients can be considered as current best practice. They are more powerful in discriminating certain object types, but do still suffer from some major drawbacks. For instance, when applying $2 \mathrm{D}$ transfer functions to modalities with a rather low signal-to-noise ratio, it is often difficult to detect boundaries and to discriminate the desired structures of interest. Furthermore, when dealing with data sets enhanced by contrast agents, it is often not possible to distinguish between bone structures and vessels filled with contrast agent, since both share rather high intensity values and a strong

\footnotetext{
*e-mail: j-s.prassni@math.uni-muenster.de

†e-mail:ropinski@math.uni-muenster.de

‡e-mail:mensmann@math.uni-muenster.de

§e-mail:khh@math.uni-muenster.de
}

gradient magnitude. Another drawback is the quite complex and non-intuitive user interface necessary for specifying these intensitygradient transfer functions.

In this paper we propose an addition to the transfer function concept along with a corresponding user interface. By applying methods already used in shape classification, we are able to define a multidimensional transfer function that takes into account the shape of a desired feature for assigning optical properties. We perform a rough, threshold-based pre-segmentation in a preprocessing step and compute the curve-skeletons for each of the resulting volumetric structures. Since curve-skeletons are well known to sufficiently describe the shape properties of 3D objects [16], we are able to derive some shape metrics for the objects, specifying the degree of membership in some predefined shape classes. These shape metrics are given by a shape descriptor, i. e., a triple (tubiness, surfaceness, blobbiness). Thus the user is able to distinguish features that are similar in terms of intensity and gradient magnitude, but do have different shapes. In order to avoid the need for intensive user interaction during the preprocessing step, we do not rely on highquality manual or semi-automatic segmentations but focus on those that can be achieved by simple windowing. As a consequence, a major challenge in the classification process for real-world data is to handle imperfections in the pre-segmentation, which may result in instabilities in the shape-skeletons and thereby disturbing shape classification. Hence, we use data preprocessing and robust classifiers to handle these issues, which would not be necessary for voxelized geometry data often used for testing skeletonization algorithms. To make our concept easily accessible, we also propose a corresponding user interface for specifying shape-based transfer functions, which is based on a continuous triangle-shaped plot showing the occurrence of the detected shape classes. The user can directly assign optical properties to these shape classes. Benefits of the presented approach are that it allows to visually separate objects that previously could only be separated by applying complex segmentation techniques, therefore requiring much less manual intervention. Furthermore, the notion of shape is very intuitive and thus the concept can also be applied without profound knowledge of the underlying algorithms, e.g., for use by domain experts.

\section{Related Work}

Transfer Functions are an essential tool for classifying volumetric data. Since 1D transfer functions cannot be used to distinguish structures having an overlapping intensity range, multidimensional transfer function techniques have been proposed. Today, the bestpractice approach considers the gradient length when assigning optical properties. Levoy [15] was the first to propose taking into account the gradient length in volume rendering. In their seminal paper on semi-automatic transfer function specification, Kindlmann and Durkin [12] show how to exploit also the second order derivative in order to semi-automatically extract boundaries from volumetric data. Although this probably forms the basis for most multidimensional transfer function approaches, the transfer function itself was still 1D and the gradient magnitude was only considered in order to modify the opacity. The method has been extended by Kniss et al. [14] to support real multidimensional transfer functions, which can be specified by the user through GUI widgets and dual-domain operations. Sereda et al. [24] also emphasize bound- 
aries with a method based on LH histograms. They identify and display surface representations in histogram space and enable the user to assign optical properties to these surfaces.

Several advanced data-centric approaches were proposed. Caban and Rheingans [3] use textural properties of volume regions instead of intensity and gradient values to control optical properties. Hadwiger et al. [8] use a pre-computed feature volume to store the results of a region growing process over a parameter domain. This allows visualization of different feature sizes without a costly re-computation of the segmentation. Correa and Ma [6] describe size transfer functions that map the relative size of features to color and opacity by utilizing scale fields for continuous representation of size. Patel et al. [18] use mean and variance of voxel intensities for a transfer function specification that is robust to noise.

The results of the transfer function comparison by Pfister et al. [19] lead to the conclusion that semi-automatic transfer function specification is the most promising approach. Therefore, we have chosen to follow this paradigm and automatically extract information in a pre-processing, which can be interactively explored by the user later on.

Our approach is most similar to the one described by Sato et al. [22]. They also support a volume classification based on shapes. However, in contrast to our technique they detect shapes, such as edges lines and blobs, by measuring multi-scale responses to $3 \mathrm{D}$ filters. Thus, their classification is performed on a per-voxel basis, whereas our approach classifies complete volumetric features, thereby allowing more intuitive user interfaces and a better understanding by domain experts. Zhang et al. [11] use inertia tensors for the classification of shapes in volumetric data sets. Their approach, however, is limited to classifying structures of a pre-defined size.

Shape Classification based on skeleton structures has a long history. In 3D, Binford's generalized cylinders [1] decompose an object into a set of elongated parts defined by sweeping a $2 \mathrm{D}$ crosssection through a 3D space curve. The concept of an axial description of shape was proposed even earlier in 2D through Blum's medial axis transform, or skeleton [2]. Pizer et al. [20] proposed a framework of stable medial representation for segmentation of objects, registration, and statistical 3D shape analysis.

Cornea et al. $[4,5]$ examined existing algorithms and introduced the concept of the hierarchical curve-skeleton as a robust method to compute increasingly detailed skeletons. Their algorithm uses a repulsive force field to extract curve-skeletons of general 3D objects from volumetric data sets, using topological characteristics such as critical points found in the resulting vector field. As the potential field is generated by charging the object's boundary, the algorithm only requires information about the object's surface voxels.

Skeletons were previously used for shape-matching, e.g., by $\mathrm{Hi}-$ laga et al. [10], but also for volume visualization. Takahashi et al. [25] automate transfer function design by extracting the topological structure of a volume data set using a skeletonization process. Reniers et al. [21] classify voxel surfaces using a 3D skeletonization method. Correa and Silver [7] use skeletons to manipulate transfer functions while they are moving along features. Cornea et al. [5] list a multitude of further uses for curve-skeletons.

\section{Classifying Shapes in Volume Data}

The goal of our approach is to construct a fuzzy shapeclassification. In contrast to shape-matching techniques which compare shapes, this classification results in several membership scores that specify how much the object matches each of the predefined shape classes. In order to allow the design of an intuitive user interface for shape classification especially for non-visualization experts, we decided to avoid the per-voxel classification performed by previous approaches [22] [11], since these require the user to analyze multi-dimensional histograms of similar complexity as the intensity-gradient transfer function space. Instead, we aimed at pre-

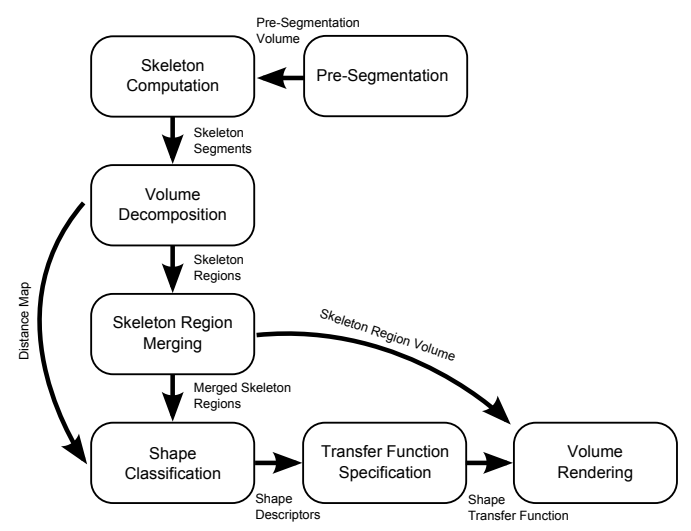

Figure 1: Workflow of our approach.

senting a manageable set of shape-classified structures to the user for the assignment of optical properties. Therefore, we have chosen a statistically motivated approach, based on curve-skeletons, that classifies structures obtained from a decomposition of the input volume. These curve-skeletons have several benefits for shapeclassification: they are invariant to translation, rotation, and scaling, and they can cope with moderate amounts of within-class deformation.

From analyzing typical volume data sets and their corresponding shape-skeletons, we have derived three independent shape classes: longitudinal/tubular, surface-like, and blobby shapes. The goal of the classification process is to assign to each voxel in the data set a score for each shape class, specifying how much the object part corresponding to the voxel matches the shape. No binary decisions are made, because volume data may contain many ambiguous shapes and the task of mapping the results of shape classification to optical properties is better left to the user in an interactive process. The overall workflow of our approach is a follows (Figure 1):

1. Creation of a pre-segmentation to obtain surface information about the objects of interest. In order to minimize the amount of user interaction that is necessary during the preprocessing step, we focus on segmentations that can be achieved by assigning intensity thresholds (windowing). In case the intensity range of the desired structures is known in advance, e.g. Hounsfield scale for CT scans, the pre-segmentation can be obtained automatically.

2. Computation of the curve-skeleton, resulting in several polygonal chains of skeleton points, the curve-skeleton segments. Additionally, we perform a normalization of the skeleton for reducing artifacts often caused by imperfect segmentations.

3. Decomposition of the pre-segmentation based on the curveskeleton segments: The volume is further divided into skeleton regions, with each region corresponding to a segment of the curve-skeleton.

4. Merging of skeleton regions. Neither the computation of the curve-skeleton nor the skeleton normalization take into account the membership of structures to the shape classes. Therefore, the skeleton regions resulting from the ad-hoc decomposition of the pre-segmentation cannot be expected to have a meaningful shape. We merge skeleton regions based on certain criteria in order to improve their classifiability.

5. Shape analysis, incorporating both curve-skeleton and volume data to assign shape scores to the merged skeleton regions, thereby constructing the shape descriptors. 

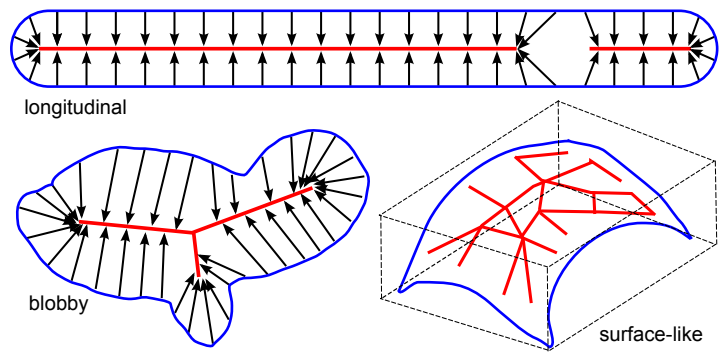

Figure 2: Typical cases for the three shape classes. The arrows illustrate the distance of each surface voxel (blue) to the skeleton segment (red). For the longitudinal shape a gap in the skeleton is displayed. The surface shape is shown along with its bounding box.

In the following subsections we first describe the shape classification scheme and then in more detail the individual steps necessary to generate the shape classification.

\subsection{Shape Classification}

The goal of the shape classification is to assign to each skeleton region generated by the previous volume decomposition a degree of membership in each of the supported shape classes. Only voxels specified in the pre-segmentation are considered, while all other voxels are considered as background voxels and ignored, setting their shape descriptor to all zero. Shape class membership is computed independently for each class, therefore further classes can be added easily. Our system supports three shape classes, namely tubiness, surfaceness, and blobbiness. In a medical context tubiness would be associated with blood vessels or elongated bones, blobbiness would be found with organs such as the heart or the kidneys. Structures with a high surfaceness could include the skullcap or a blade-bone. The three shape classes can also be interpreted as a measure of dimensionality, i.e., a shape with a onedimensional elongation gets a larger tubiness value, whereas a more three-dimensional shape gets a larger blobbiness value.

\subsubsection{Tubiness}

We define an elongated region having a circular cross-section of constant diameter as a perfectly tubular structure. In theory, such structures feature a curve-skeleton that runs through the center of each cross-section, and each of the region's surface points has the same minimal distance to the curve-skeleton: the radius of the tube. Therefore, we define the tubiness $\tau$ of a volumetric region $R$ as the inverse of the standard deviation of its surface voxels' minimal skeleton distances, i. e.,

$$
\tau(R):=\frac{1}{\max \left(\sqrt{\frac{1}{\partial R \mid} \sum_{\vec{v} \in \partial R}(D(\vec{v})-\bar{d})^{2}}, 1\right)} \in[0 ; 1],
$$

where $\partial R$ is the set of surface voxels of $R, D$ is the distance map computed during the skeleton region growing, and $\bar{d}$ is the mean skeleton distance of all $v \in \partial R$. In contrast, both surfaces and blobby regions usually feature a significantly higher standard deviation, as Figure 2 illustrates. Note that the tubiness classifier is not restricted to cylindric shapes but yields similar classification results for curved longitudinal shapes such as rib bones.

The reliability of the tubiness classifier depends to some degree on the quality of the computed curve-skeletons, since especially an incomplete, discontinuous skeleton segment boosts the standard deviation of a tubinal region's surface-to-skeleton distances. By connecting adjacent skeleton segments during the skeleton normalization we were able to handle this issue in most cases. Only highly degenerated tubular regions where only a small fraction is represented correctly by the skeleton may be misclassified.

\subsubsection{Surfaceness}

A surface can be either planar or folded in space. In the first case, the object's minimal oriented bounding box has a very small extent in one space direction compared to the extent in the two other space directions. We compute the minimal oriented bounding box for each region by applying Har-Peled's [9] technique. The planarity of a region is then proportional to the ratio of the bounding box's second shortest side length $b_{m}$ and its shortest side length $b_{s}$. According to our notion of planarity, we define regions with $b_{m} \geq 10 \cdot b_{s}$ to be maximal flat and regions with $b_{m} \leq 5 \cdot b_{s}$ to be not flat at all and apply a linear transition between these extrema:

$$
\operatorname{plan}(R):=\operatorname{clamp}\left(\frac{1}{5} \frac{b_{m}(R)}{b_{s}(R)}-1,0,1\right) \in[0 ; 1]
$$

In case of a curved surface, the bounding box criterion does not work. Therefore, we additionally compute the convexity of each shape by selecting an arbitrary set of pairs of surface points and determining for each line segment formed by a point pair the fraction that runs inside the shape. The convexity $\operatorname{conv}(R)$ is then the average of these fractions. Since curved surfaces occupy only a small part of their bounding box's volume as depicted in Figure 2, most parts of the line segments between surface points are lying outside the region. Note that the sum of the planarity measure and the convexity measure in isolation is not an appropriate surfaceness classifier, since curved longitudinal shapes might have both planar bounding boxes as well as a low convexity. Therefore, their sum has to be weighted by the inverse tubiness score:

$$
\operatorname{surf}(R):=(\operatorname{plan}(R)+\operatorname{conv}(R)) \cdot(1-\tau(R)) \in[0 ; 2]
$$

\subsubsection{Blobbiness}

The goal of the blobbiness classifier is to detect volumetric regions that humans intuitively regard as "compact". Though it is hard to give a precise definition of blobbiness, one can certainly say that a sphere or a cube are blobby objects, whereas tubes or planar structures are less so. Furthermore, a suitable blobbiness classifier should classify an ellipse or a cuboid less blobby than a sphere or a cube. One possibility could be the examination of the ratio of surface to volume of a given region, since it can be shown that a sphere has minimum surface for a given volume and that a cube has a smaller surface than any non-cubic cuboid of the same volume. But although the surface-to-volume ratio seems to be an appropriate indicator for the blobbiness of analytical structures, it is less useful for the classification of volumetric data sets, which often contain regions with rough, imperfect boundaries. These jagged regions exhibit a larger surface (i. e., number of surface voxels) than an analytical object of the same global shape, resulting in a misleading surface-to-volume ratio.

Therefore, we follow a statistically motivated approach focussing on the spatial distribution of a region's voxels instead of its surface. We interpret the volumetric region $R$ as a probability distribution and compute its second central moment about the mean, also called variance, i. e.,

$$
\sigma^{2}(R):=\sum_{\vec{r} \in R}|\vec{r}-\bar{m}|^{2}
$$

where $\vec{r}$ are the coordinates of a voxel $\in R$ and $\bar{m}$ is the region's center of mass, i. e.,

$$
\bar{m}=\frac{1}{|R|} \sum_{\vec{r} \in R} \vec{r}
$$

From an intuitive point of view, the variance measures to what extent a region's mass is centered around its center of mass, in other words the variance determines the compactness of an object. More precisely, a sphere is the shape with minimal variance for a given 
volume, since no volume element can be moved any closer to the center of mass in order to reduce the variance. Therefore, we consider the variance of a volumetric region an appropriate measure of its blobbiness. However, the variance is heavily influenced by the size of an object, as an object with larger volume needs to occupy a larger region, which increases its variance. In contrast, we are interested in a size-independent measure of blobbiness, because we generally consider shape and size as independent concepts. We achieve this by expressing a region's variance relatively to the variance of a sphere with the same volume. First, we express a sphere's radius $r$ as a function of its volume vol:

$$
\operatorname{vol}(r)=\frac{4}{3} \pi r^{3} \Leftrightarrow r(v o l)=\left(\frac{3}{4 \pi} v o l\right)^{1 / 3}
$$

Second, we calculate the variance $\sigma_{r_{0}}^{2}$ of a sphere with radius $r_{0}$, which is centered around the origin, by integrating over all spheresurfaces with radius $\leq r_{0}$ :

$$
\sigma_{r_{0}}^{2}=\int_{0}^{r_{0}}\left(4 \pi r^{2}\right) r^{2} d r=\frac{4}{5} \pi r_{0}^{5}
$$

Inserting Eqn. (6) into Eqn. (7) yields the variance of a sphere with volume vol:

$$
\sigma_{s p h}^{2}(v o l)=\frac{4}{5} \pi\left(\frac{3}{4 \pi} v o l\right)^{5 / 3}
$$

Now, we are able to express a region's variance in terms of the variance of a sphere of the same size. Hence, we define the blobbiness of a region $R$ as:

$$
\operatorname{blob}(R):=\frac{\sigma_{s p h}^{2}(|R|)}{\sigma^{2}(R)} \in[0 ; 1]
$$

\subsection{Pre-segmentation}

As such, volumetric data does not have a notion of shape. Some semantic information needs to be added to the intensity values associated with the voxels in order to be able to define shapes, mainly by specifying where object boundaries are located, i. e., which voxels are part of an object's surface. For typical volumetric data sets, this decision can be easy, as it is the case with the sharp contrast between a metallic object and surrounding air in an industrial CT scan, or more complicated such as for an inner organ surrounded by tissue of similar intensity in a medical context. Note, however, that for shape analysis not a full segmentation is required, only surfaces need to be specified. For example, two or more objects which would be placed in individual segments for a full segmentation can be placed in the same segment when only their surface is important. Splitting up this pre-segmentation into multiple sub-segments corresponding to individual objects is left to the subsequent shape classification. Hence, instead of a full-blown segmentation technique a much simpler method such as windowing can be applied to acquire a rough approximation of object surfaces inside the volume data.

\subsection{Curve-Skeleton Computation}

A major issue with skeleton computation is stability. Even small changes in the object data, as caused by noise, can have a great influence on the resulting curve-skeleton. This is less of a problem for our classification scheme, as we are analyzing each skeleton segment by itself, and therefore we incorporate more information with a statistical approach that is less likely to be influenced by local stability issues. Cornea et al. [4] have evaluated several methods for computing curve-skeletons, noting that the potential field method results in the cleanest and smoothest curve-skeletons. We were especially interested in smooth skeletons of low complexity, because

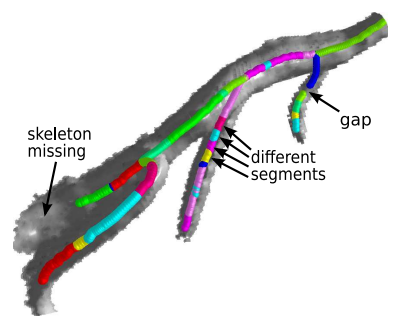

Figure 3: Close-up of curve-skeleton in an angiography data set prior to normalization. Pre-segmentation errors can lead to gaps in the skeleton, as well as missing skeletons for entire parts of the volume. The skeleton segments are shown with alternating colors.

heavily branching skeletons would cause problems for the volume decomposition and merging step. Therefore, we have chosen the potential field method, although it is the slowest of the examined techniques, but running time of the preprocessing is not a main issue for our use case.

Our setup uses Cornea's pfSkel application [5] that computes the potential-based vector field of the object specified in the input presegmentation to construct the segments of the 3D curve-skeleton. The algorithm requires a binary volume as input to specify surface voxels. It outputs the generated curve-skeleton as multiple skeleton segments, each consisting of a polygonal chain of seed points. Additionally, information about critical points and high divergence points are given, but these are not used for our technique. To prevent cavities inside the pre-segmentation, caused by noise or other artifacts, from disturbing computation of the skeletons, we add extra layers of voxels at the surface of objects. While this can have the effect of smoothing the object and may lead to unwanted merging of adjacent features, it worked out to remove some noise with the relatively high resolution data sets we used. The only further relevant parameters are field strength, for which we chose a low value of 4 to get a minimal complexity skeleton, and percentage of high divergence points to use, for which we stayed with the default $20 \%$ for all examined data sets.

Though our statistically motivated classification approach is relatively robust towards imperfections of the curve-skeleton, there are still some computation artifacts and properties inherent to curveskeletons that cause problems during the subsequent steps. Therefore, we perform three basic post-processing steps on the computed skeleton, which we call skeleton normalization:

1. Thin longitudinal structures such as vessels often exhibit gaps in the skeleton as depicted in Figure 3. Since these gaps hamper the applicability of the tubiness classifier, we close them by connecting each end node of the skeleton graph with its next neighbored skeleton point outside the end node's skeleton segment, if the connecting line lies completely within the pre-segmented object.

2. When a blobby shape is passing into a tubular structure, the skeleton segment running through both is not necessarily split up at the border region between the two shapes. It would be impossible to classify the blobby shape in isolation as the skeleton region corresponding to the skeleton segment also contains the respective part of the tubular structure. Moreover, in case the blobby part of the region is dominated by the tubular one, the region would not even be recognized as blob. Therefore, we split the curve-skeleton into segments not exceeding a certain length as shown in Figure 4. For the data sets with sizes of $256^{3}$ to $512^{3}$ we examined, length thresholds of five to ten voxels gave good results. Due to the curve-skeleton decomposition, however, the subsequent region merging step gains in importance. 3. Degenerated skeleton branches shorter than the length threshold are discarded (pruning) as these may cause problems during the region merging. 


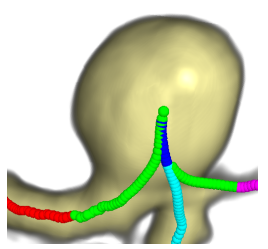

(a)

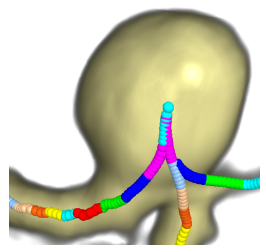

(b)
Figure 4: Comparison of the curve-skeleton of an aneurysm blob before (a) and after (b) the splitting operation. Without splitting the left green segment ranges from the blob deep into the left vessel preventing a proper classification of the blob.

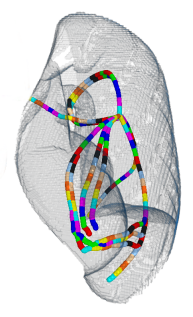

(a)

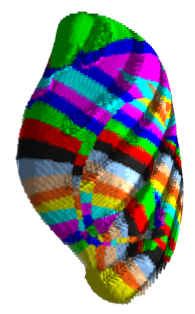

(b)

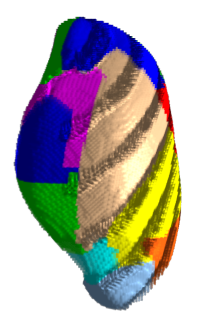

(c)
Figure 5: Blob merging demonstrated on one lung of the NCAT phantom [23]. (a) shows the curve-skeleton after normalization, (b) the corresponding skeleton regions, (c) the regions after fusion.

\subsection{Volume Decomposition}

The rough pre-segmentation consisting of a few very large segments is not suitable for any shape classification. Therefore, we have to further decompose the pre-segmented volume into smaller segments, which more likely exhibit pronounced shapes, before applying the shape classifiers. This decomposition is based on the previously computed skeleton. In a curve-skeleton as returned by the potential field algorithm, however, there is no connection between the skeleton segments and individual voxels in the data set. Thus, we perform a distance transform in order to compute the distance of each voxel to its nearest skeleton segment and assign the voxel to this segment. The distance transform is done by simultaneous region growing, where each of the skeleton segments is chosen as a separate seed. In contrast to the hierarchical mesh decomposition presented by Cornea et al. [5] this includes not only surface voxels but all voxels associated with a skeleton segment, forming the skeleton region. The region growing process assigns each voxel that was initially part of the pre-segmentation to such a skeleton region. The distance map containing the minimal distance of each voxel to its next skeleton segment is used in subsequent steps.

\subsection{Merging of Skeleton Regions}

The volume decomposition outputs a heavily over-segmented data set, since each segment of the normalized curve-skeleton is assigned a skeleton region. Although the over-segmentation is necessary in order to make sure that no region of the decomposed volume contains structures of different shape classes, it also causes the skeleton regions to loose their initial shape property. For instance, the splitting of a vessel causes an originally tubular region to become blobby. Therefore, the fusion of skeleton regions into classifiable units is a crucial step of the classification process. One might get the impression that volume decomposition and region merging are inverse operations. However, while the volume decomposition is based on the geometry of the curve-skeleton, the fusion process also takes into account the shape properties of skeleton regions. The merging steps are as follows:

- First of all, very small regions are merged with the neighbor region with which they share the largest part of their surface, since those regions cannot be expected to have any meaningful shape. In order to avoid the necessity to manually specify a minimal segment size, we define each segment, whose size deviates down from the mean segment size by more than two times the standard deviation, as too small.

- Tube merging. In order to allow a reliable classification of tubes, split tubular structures have to be merged as far as possible, while preventing erroneous fusions with blobs or surface-like structures. We have chosen the tubiness score as merge criterion. More precisely, we fuse all pairs of neighbored regions that both as well as the merge result have a tubiness score above a certain threshold. Note that the tubiness classifier itself is not affected by the splitting of tubular structures. Instead, the misclassification is caused by the fact that the splitting significantly increases the blobbiness score. Therefore, it is reasonable to base the tube merging on the tubiness classifier. In addition, the merging stops at skeleton branches, i.e. two regions are not merged, if the connection point of their skeleton segments is the origin of a third segment. In our examinations, a tubiness threshold of around 0.8 turned out to be the best choice.

- Blob merging. The curve-skeleton of blobby structures is usually heavily branching, which in combination with the skeleton splitting leads to many thin regions that would not be classified as blobby as illustrated for one lung of the NCAT phantom in Figure 5 (a). Such regions have in common that they share a much larger border with other regions (inner surface) than with the background (outer surface). We merge regions with a high inner-surface-to-outer-surface ratio. As a result, split up blobby structures are usually not fused into a single region, but the remaining regions have regained a blobby shape and can thus be classified correctly, as visible in Figure 5 (c).

- Quality-based merging. Since one main objective of the previous fusion steps is not to erroneously merge regions of different shape classes, these steps might fail on imperfect skeleton segments or object surfaces. For instance, a vessel region with a rough surface due to artifacts in the pre-segmentation may have a low tubiness score and might therefore not be merged with neighbored vessel regions. The last fusion step copes with these situations by trying to improve the clarity of the classification: two regions are merged when the classification of the resulting shape is less ambiguous than the input regions' classifications. We define the ambiguity of a region $R$ as the quotient of its lowest and highest classification score:

$$
\operatorname{amb}(R):=\frac{\min (\tau(R), \operatorname{surf}(R), \operatorname{blob}(R))}{\max (\tau(R), \operatorname{surf}(R), \operatorname{blob}(R))}
$$

\section{User Interface for Shape Transfer Functions}

To be able to intuitively assign optical properties to certain shape classes as identified by our approach, a sufficient user interface is required. In this section, we briefly describe such a user interface concept. It allows us to represent the 4-dimensional transfer function space, given by the classifiers tubiness, surfaceness and blobbiness as well as the intensity values, in a comprehensible way. For simplicity, we consider the shape-based transfer function assignment as a two-stage process, where the user first constrains the shapes to be visualized before defining the desired intensity range. 


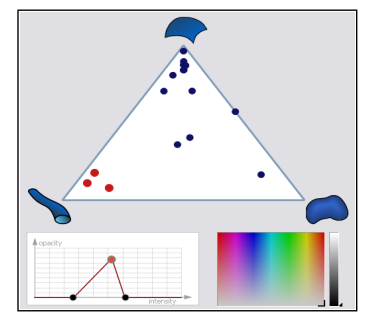

Figure 6: Shape-selection widget. Each shape is represented by a marker whose position depicts its degree of membership in the three shape classes. The user has selected three tubinal shapes (red).

\begin{tabular}{|l|c|c|c|}
\hline data set & resolution & $t_{\text {skel }}$ & $t_{\text {class }}$ \\
\hline angiography 1/2 & $256^{3}$ & $210 / 176$ & $13 / 9$ \\
mouse (cardiac) & $225 \times 178 \times 256$ & 512 & 7 \\
mouse (torso) & $360 \times 290 \times 400$ & 1930 & 27 \\
\hline
\end{tabular}

Table 1: Statistics for the data sets presented in the results section. Given are the data set resolution, the times for computation of the curve-skeleton and for the classification (in seconds).

The normalization of the shape classifiers makes them comparable and allows the user to intuitively constrain the visualization to certain shape classes. This normalization gives us the opportunity to use an equilateral triangular shape selection widget based on barycentric coordinates (see Figure 6), similar to the one proposed by Kindlmann and Weinstein [13] for DTI data. Each corner of the triangle represents one of the three shape classes. For each computed shape segment we place a marker inside the triangle in such a way that the marker's position indicates the likelihood that the shape class is best represented by one of the three basic shapes. For the placement of each marker we use the barycentric coordinates defined by the three normalized shape classifiers. Then a marker is placed closer to those corners of the triangle that represent the shape classes the identified segment is best classified as. Thus, in the first processing step, the user is able to select an arbitrary number of markers to constrain the shape classes to be visualized. In the second step, a conventional 1D transfer function is used to assign optical properties to the selected shape classes. For the examination of data sets with no planar structures, the surfaceness class can be omitted collapsing the triangle to a line.

\section{Results}

We have integrated shape-based transfer functions into the Voreen volume rendering engine [17] that implements GPU-accelerated ray casting using OpenGL fragment shaders and have tested our technique with several synthetic as well as real-world data sets, mostly from the medical domain. All pre-segmentations of the data sets presented in this section are a result of windowing. We stayed with our default merging parameters of 0.8 for the tubiness threshold and 0.1 for the maximum inner-surface-to-outer-surface ratio, while adjusting the maximum skeleton segment length to the sizes of the data sets. User interaction was only required for the specification of the intensity range used for the pre-segmentation and for the assignment of transfer functions to the shapes, while the intermediate classification process runs automatically. Preprocessing times for the different data sets are given in Table 1. All tests were conducted on a system equipped with an Intel Core 2 Quad Q9550 CPU and an NVIDIA GeForce GTX 280 graphics board.

Figure 7 depicts the single steps of the classification process for an angiography data set. The volume decomposition based on the normalized curve-skeleton yields a heavily over-segmented volume (Figure 7(b)). Therefore, the initial skeleton regions mostly lack pronounced shapes and exhibit rather random shape scores, which are distributed almost uniformly over the whole spectrum. The region merging fuses the more than 200 initial regions to nine final features with pronounced shapes (Figure 7(c)): The aneurysm is correctly classified as an isolated blob, while the three large vessel regions (blue, green, ocher) are represented correctly by the leftmost shape cluster in the user interface. The ambiguity of the five remaining vessel shapes is caused by imperfect skeleton segments, which lower the tubiness score of their regions. However, these regions are still clearly distinguishable from the aneurysm.

Figure 8 shows the classification results of two angiography data sets. In both cases the blobby aneurysms could be easily isolated from the tubular vessels. We omitted the surfaceness classifier for all angiography data sets, since they do not contain such structures.

In Figure 9 we applied our technique to the CT scan of a mouse heart. Though the shape classification is less clear than for the angiographies, the two shape clusters corresponding to the blobby heart structures and the vessels, respectively, can be easily identified in the user interface. Furthermore, the vessels are mostly correctly separated from the heart, though a slight misclassification is visible in the third rendering where parts of the vessels have been classified as blobby. This is due to the fact that these vessel regions touch the heart structures and are merged with them.

Figure 10 shows the shape-classification of a CT scan of a mouse lying on a bed. This case is interesting for several reasons. Since the heart and vessels are filled with contrast agents, their intensity range overlaps with the bones' range and can therefore not be separated by a conventional 1D transfer function, a typical situation in contrast-enhanced CT scans. Furthermore, the bed as a surface-like structure also shares this intensity range as visible in 10 (a). The shape-classification shown in 10 (b) allows a separation of the heart from the bones and vessels as well as the bed. We consider the classification of the elbows to be correct, since though they are part of a longitudinal structure the elbows themselves are of blobby shape. The blobby classification of parts of the shoulder bones, however, is an error that is caused by gaps in the skeleton in that regions. In 10 (c) the classification has been manually refined through the user interface by removing the blobby structures outside the heart.

\section{Current Limitations and Future Work}

A principal limitation of our approach is the dependency on a proper masking of the volumetric structures that are to be shapeclassified. While such a masking might be tedious in the general case, creating a sufficiently precise pre-segmentation for volumetric scans with contrast agents, which are a typical use-case for volume visualization in the medical domain, is possible with little effort by windowing.

If an adequate masking is provided, errors or ambiguities in the shape classification are mainly caused by incomplete merging operations, since small regions are less likely to exhibit a pronounced shape than larger ones. On the other hand, lowering the merge barrier increases the risk of erroneously fusing shapes of different classes. We want to investigate whether a more global merge strategy, which does not focus on the local neighborhood of regions but rather tries to increase the overall clarity of the classification, can improve this issue. Furthermore, we currently only use information from level 0 of the skeleton hierarchy, the "core skeleton", while incorporating low divergence points and high curvature points added in levels 1 and 2 might help to refine the region merging.

A further issue is the significant time consumption of the shape classification and especially the skeleton computation, which do currently not allow an interactive parameter tuning. While we are positive that a more efficient implementation can achieve interactive results for the merging and classification steps, it should also be investigated whether a simpler skeletonization algorithm could possibly give similar results while having less demanding runtime requirements compared to the potential field technique. 


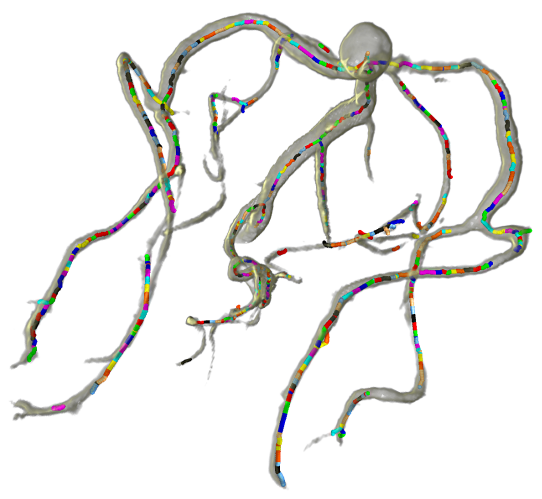

(a)

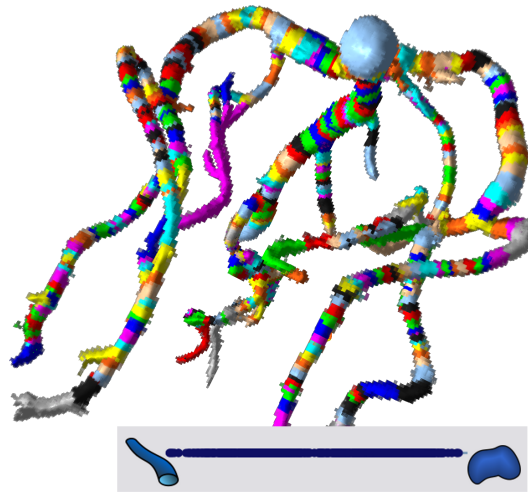

(b)

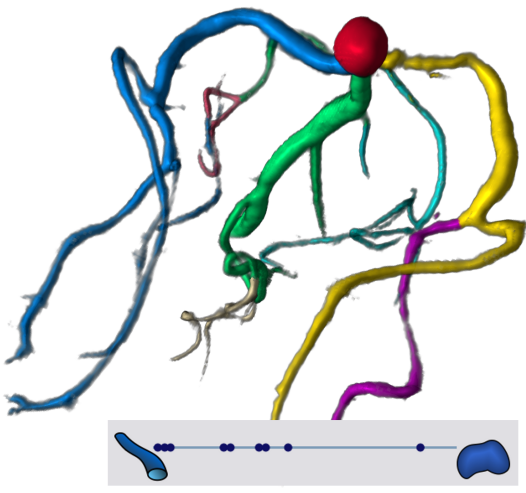

(c)

Figure 7: Classification workflow for the angiography data set shown in Figure 8(b). Subfigure (a) displays the normalized curve-skeleton, while (b) and (c) present the skeleton regions before and after merging along with the corresponding shape distributions.

\section{Conclusion}

When exploring volumetric data, the user is rather interested in visually inspecting features than extracting them. We have introduced shape-based transfer functions in order to blur the border between simple but limited classification and powerful but costly segmentation techniques. In contrast to previous approaches towards shape classification in volume data, which perform a voxel level classification, the proposed technique does not require the user to interpret complex histograms but provides him/her with a manageable set of shape-classified volumetric features and offers an intuitive interface for the assignment of optical properties. Since we consider shape, texture and size as independent properties of volumetric features, we believe that a combination of our approach with texturebased [3] and size-based [6] [8] classification schemes might be worth investigating.

\section{ACKNOWLEDGEMENTS}

This work was partly supported by grants from the Deutsche Forschungsgemeinschaft (DFG), SFB 656 MoBil Münster, Germany (project Z1). The presented concepts have been integrated into the Voreen volume rendering engine (www.voreen.org).

\section{REFERENCES}

[1] T. Binford. Visual perception by computer. In IEEE Conference on Systems and Control, 1971.

[2] H. Blum. Biological shape and visual science. Theoretical Biology, 38:205-287, 1973

[3] J. J. Caban and P. Rheingans. Texture-based transfer functions for direct volume rendering. IEEE TVCG, 14(6):1364-1371, 2008.

[4] N. D. Cornea, D. Silver, and P. Min. Curve-skeleton properties, applications, and algorithms. IEEE TVCG, 13(3):530-548, 2007.

[5] N. D. Cornea, D. Silver, X. Yuan, and R. Balasubramanian. Computing hierarchical curve-skeletons of 3D objects. The Visual Computer, 21(11):945-955, 2005.

[6] C. D. Correa and K.-L. Ma. Size-based transfer functions: A new volume exploration technique. IEEE TVCG, 14(6):1380-1387, 2008.

[7] C. D. Correa and D. Silver. Dataset traversal with motion-controlled transfer functions. In IEEE Visualization 2005, pages 359-366, 2005.

[8] M. Hadwiger, F. Laura, C. Rezk-Salama, T. Höllt, G. Geier, and T. Pabel. Interactive volume exploration for feature detection and quantification in industrial CT data. IEEE TVCG, 14(6):1507-1514, 2008.

[9] S. Har-Peled. A practical approach for computing the diameter of a point set. In SCG '01: Proceedings of the 17th annual symposium on Computational geometry, pages 177-186. ACM, 2001.

[10] M. Hilaga, Y. Shinagawa, T. Kohmura, and T. L. Kunii. Topology matching for fully automatic similarity estimation of 3D shapes. In
SIGGRAPH '01: Proc. of the 28th annual conference on Computer graphics and interactive techniques, pages 203-212, 2001.

[11] J. Z. Huawei Hou, Jizhou Sun. Moment based transfer function design for volume rendering. In Grid and Cooperative Computing: Second International Workshop, pages 215-218, 2003.

[12] G. Kindlmann and J. W. Durkin. Semi-automatic generation of transfer functions for direct volume rendering. In VVS '98: Proc. of the 1998 IEEE Symposium on Volume Visualization, pages 79-86, 1998.

[13] G. Kindlmann and D. Weinstein. Hue-balls and lit-tensors for direct volume rendering of diffusion tensor fields. In IEEE Visualization 99 Proceedings, pages 183-189, 1999.

[14] J. Kniss, G. Kindlmann, and C. Hansen. Multidimensional transfer functions for interactive volume rendering. IEEE Transactions on $\mathrm{Vi}$ sualization and Computer Graphics, 8(3):270-285, 2002.

[15] M. Levoy. Display of surfaces from volume data. IEEE Computer Graphics and Applications, 8(3):29-37, 1988.

[16] D. Macrini, K. Siddiqi, and S. Dickinson. From skeletons to bone graphs: Medial abstraction for object recognition. IEEE Computer Society Conf. on Computer Vision and Pattern Recognition, 0:1-8, 2008.

[17] J. Meyer-Spradow, T. Ropinski, J. Mensmann, and K. Hinrichs. Voreen: A rapid-prototyping environment for ray-casting-based volume visualizations. IEEE Computer Graphics and Applications, 29(6):6-13, 2009.

[18] D. Patel, M. Haidacher, J.-P. Balabanian, and M. E. Gröller. Moment curves. In Proc. of the IEEE Pacific Visualization Symposium 2009, pages 201-208, 2009.

[19] H. Pfister, B. Lorensen, C. Bajaj, G. Kindlmann, W. Schroeder, L. S. Avila, K. Martin, R. Machiraju, and J. Lee. The transfer function bake-off. IEEE Computer Graphics and Appl., 21(3):16-22, 2001.

[20] S. M. Pizer, G. Gerig, S. C. Joshi, and S. R. Aylward. Multiscale medial shape-based analysis of image objects. Proceedings of the IEEE, 91(10):1670-1679, 2003.

[21] D. Reniers, A. Jalba, and A. Telea. Robust classification and analysis of anatomical surfaces using 3D skeletons. In Eurographics Workshop on Visual Computing for Biomedicine, 2008.

[22] Y. Sato, C.-F. Westin, A. Bhalerao, S. Nakajima, N. Shiraga, and $\mathrm{S}$. Tamura. Tissue classification based on 3D local intensity structures for volume rendering. IEEE TVCG, 6(2):160-180, 2000.

[23] W. P. Segars. Development and Application of the New Dynamic NURBS-based Cardiac-Torso (NCAT) Phantom. PhD thesis, University of North Carolina at Chapel Hill, 2001.

[24] P. Sereda, A. Vilanova Bartroli, I. W. O. Serlie, and F. A. Gerritsen. Visualization of boundaries in volumetric data sets using LH histograms. IEEE Transactions on Visualization and Computer Graphics, 12(2):208-218, 2006

[25] S. Takahashi, Y. Takeshima, and I. Fujishiro. Topological volume skeletonization and its application to transfer function design. Graph ical Models, 66(1):24-49, 2004. 


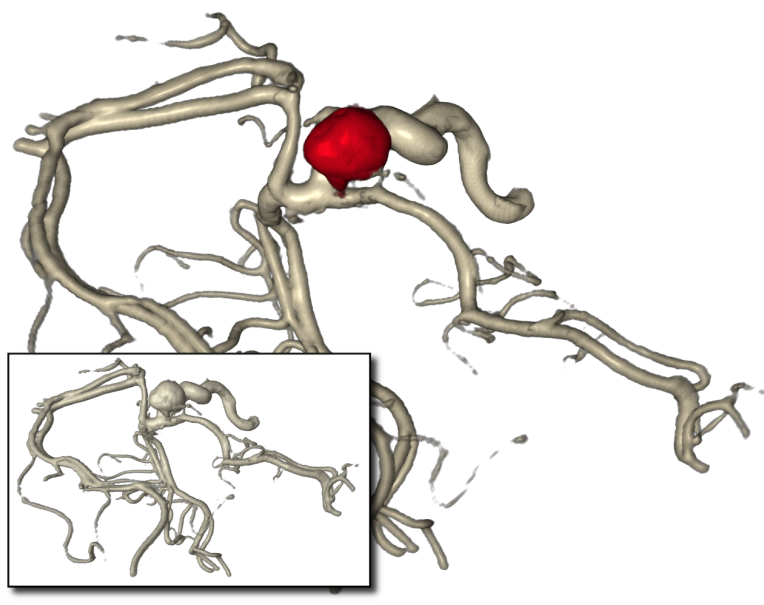

(a)

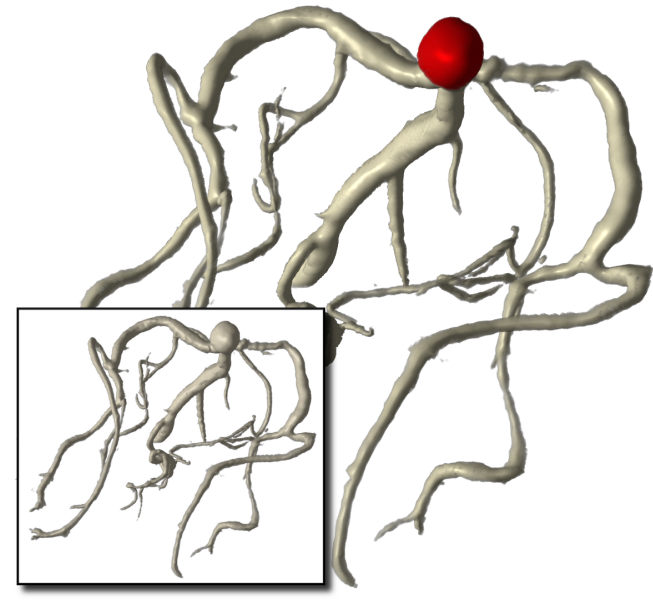

(b)

Figure 8: Shape-classified volume renderings of two angiography data sets, each containing an aneurysm. The thumbnails show renderings generated with a conventional $1 \mathrm{D}$ transfer function, for comparison.

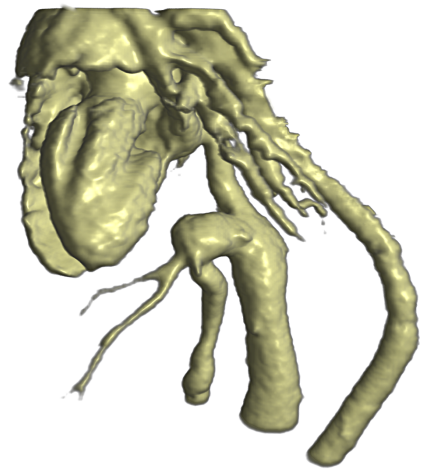

(a)

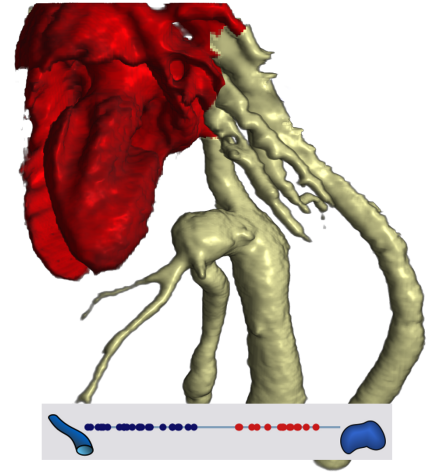

(b)

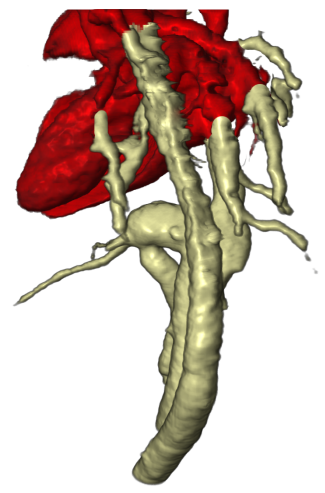

(c)

Figure 9: Application of the proposed classification technique to a CT scan of a mouse heart. (b) and (c) show renderings of the classification result from two perspectives along with the corresponding shape distribution. The red-labeled heart structures correspond to the shapes selected in the user interface. The rendering in (a) was generated with a conventional 1D transfer function.
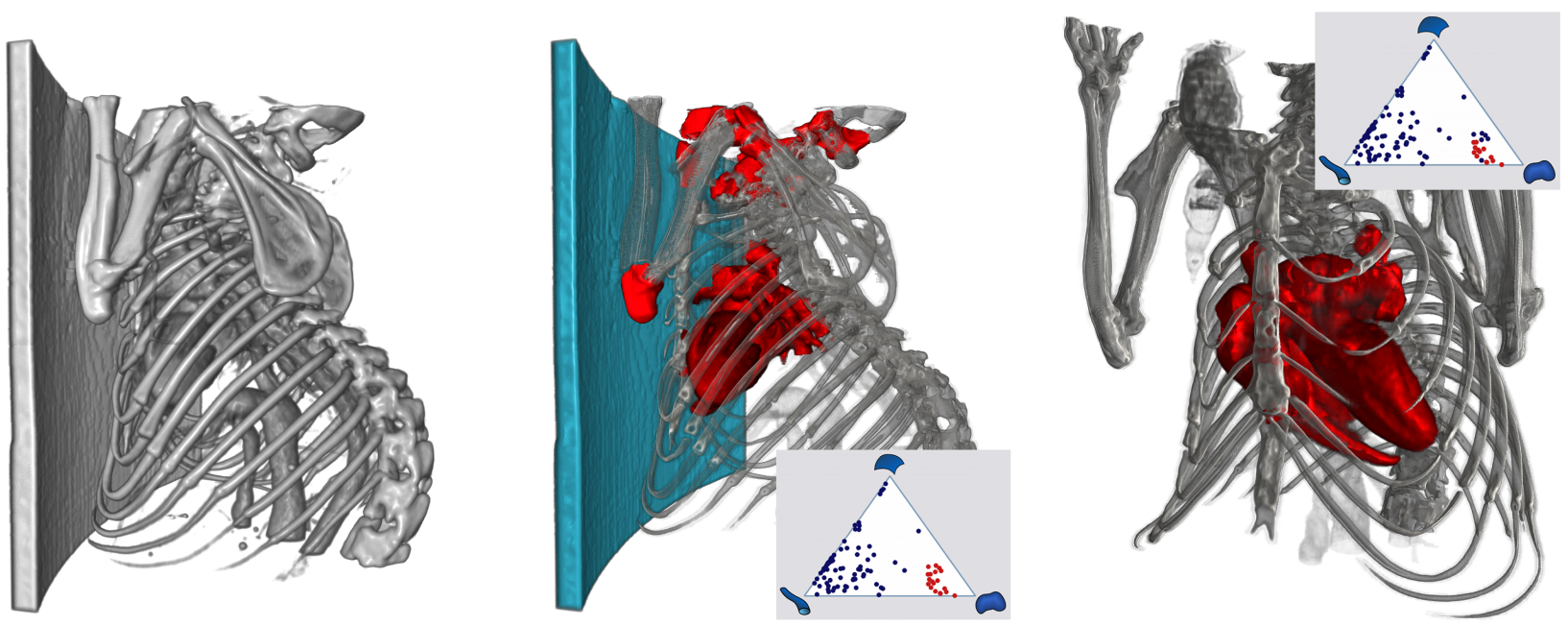

Figure 10: Shape-classified CT scan of a mouse lying on a bed. (a) was rendered with a conventional 1D transfer function. In (b) the bones/vessels are rendered semi-transparently, while the heart and the bed have been colored independently. The blobby structures (red) have been selected in the user interface. (c) shows a further refined classification where the blobby features outside the heart have been selected in the user interface in order to assign the same optical properties that have been chosen for the bones/vessels. 